\title{
Long term follow up of 69 patients treated for optic pathway tumours before the chemotherapy
}

era

C Cappelli, J Grill, M Raquin, A Pierre-Kahn, A Lellouch-Tubiana, M-J Terrier-Lacombe, J-L Habrand, D Couanet, R Brauner, D Rodriguez, O Hartmann, C Kalifa

Department of Pediatrics, Gustave Roussy Institute, 39 rue Camille Desmoulins, 94805 Villejuif, Cedex, France

C Cappelli

J Grill

$M$ Raquin

O Hartmann

C Kalifa

Department of Pathology, Gustave Roussy Institute M-J Terrier-Lacombe

Department of Radiation Therapy, Gustave Roussy Institute

J-L Habrand

Department of Radiology, Gustave Roussy Institute D Couanet

Department of Neurosurgery, Hôpital Necker-Enfants Malades, 75015 Paris, France

A Pierre-Kahn

Department of Pathology, Hôpital Necker-Enfants

Malades

A Lellouch-Tubiana

Department of Endocrinology, Hôpital Necker-Enfants

Malades

R Brauner

Department of Neuropediatrics, Hôpital Saint-Vincent de Paul, 75674 Paris Cedex 14, France

D Rodriguez

Correspondence to: Dr Grill.

e-mail: jgrill@igr.fr

Accepted 8 May 1998

\begin{abstract}
Aim-To analyse the long term results of conservative management with radiotherapy in patients with optic pathway tumours.

Design-All 69 patients were symptomatic at diagnosis and most neoplasms involved the optic chiasm and hypothalamus.

Results-At 10 years, overall survival and progression free survival were $83 \%$ and $65.5 \%$, respectively. After radiotherapy, vision improved in 18 patients and remained stable in 29 other patients. Cerebrovascular complications occurred in nine of 53 patients treated with radiotherapy after a median interval of two and a half years. These complications were five times more frequent in patients with neurofibromatosis type 1 (NF1). Severe intellectual disabilities were present in 18 children, most of whom underwent irradiation at a very young age (median age, 4 years).

Implications-Radiotherapy is a valuable treatment in terms of tumour response, visual outcome, and progression free survival. However, in young children and in patients with NF1, major sequelae are encountered and new treatment strategies should be proposed for these patients. (Arch Dis Child 1998;79:334-338)
\end{abstract}

Keywords: optic pathway tumours, neurofibromatosis type 1 ; radiotherapy; long term follow up

Optic pathway tumours account for $5 \%$ of childhood intracranial tumours. ${ }^{1}$ Although these tumours are usually low grade astrocytomas, their natural history can be extremely erratic, ranging from long term stabilisation to progressive visual and neurological deterioration, culminating in death. ${ }^{2}$ Some patients might even present with metastasis at diagnosis $^{3}$ or during the course of their disease, ${ }^{4}$ as demonstrated by sophisticated radiological techniques, such as magnetic resonance imaging (MRI). Because of this erratic behaviour, there is no consensus about the most appropriate management for these tumours. ${ }^{56}$ Surgery might be considered the best treatment for an optic nerve tumour of a blind eye, but chiasmatic and hypothalamic disease might require a totally different approach. With radiotherapy alone, or combined with surgery, progression free survival rates can attain $90 \%$ at five years. ${ }^{78}$ Moreover, most of the patients will benefit from radiotherapy for the preservation of visual function and acuity. ${ }^{89}$ However, survival rates decrease over time as a result of late relapses or complications. ${ }^{27}$ In recent years, chemotherapy has emerged as an interesting therapeutic tool for the management of optic pathway gliomas. ${ }^{10-14}$ The respective role and toxicity of these treatments need to be evaluated carefully before guidelines can be proposed. To clarify the usefulness and drawbacks of radiotherapy, we reviewed a large series of children treated in our institution before the chemotherapy era and after the availability of computed tomography (CT). Our study spans a 12 year period between 1980 and 1992.

\section{Patients and methods}

PATIENTS

All children treated for an optic pathway tumour after 1980 at the Institut Gustave Roussy were enrolled in the study provided that their initial treatment did not include chemotherapy. After review, 69 patients were included. Six children were excluded because they had received chemotherapy before radiation or because of insufficient radiological documents. All patients were referred to our institute for complementary treatment of either progressive or life threatening tumours. Clinical data were obtained from the patients' files. Ophthalmological evaluation included a fundus examination, visual field assessment, and measurement of visual acuity. Endocrinological evaluation was performed at various stages of the treatment either before or after radiotherapy and included standard procedures described elsewhere. ${ }^{15}$ Neurofibromatosis type 1 (NF1) was diagnosed either initially or during the course of the disease using standard clinical criteria. ${ }^{16} \mathrm{CT}$ scans or MRI of the tumour were mandatory at diagnosis and at follow up. Children were included in this review regardless of the extent of the optic pathway tumour, which was classified by involved site according to Dodge classification: type I, a single optic nerve alone; type II, the optic chiasm and one or two optic nerves; type III, involvement of the hypothalamus or adjacent structures. ${ }^{17}$

\section{EVALUATION OF RESPONSE}

Response to radiotherapy was evaluated clinically and by ophthalmological examination, and radiologically by repeated CT scans or MRI. Radiological classification of responses followed International Society of Pediatric Oncology (SIOP) guidelines. ${ }^{18}$ 
Table 1 Clinical presentation

\begin{tabular}{lr}
\hline Neurofibromatosis type 1 (NF1) & 40 of 69 \\
Family history of NF1 & 19 of 40 \\
Raised intracranial pressure & 17 of 69 \\
Impaired psychomotor development & 10 of 69 \\
Localised motor deficit & 8 of 69 \\
Seizures & 6 of 69 \\
Diabetes insipidus & 2 of 69 \\
Early puberty & 6 of 69 \\
Russel syndrome (diencephalic cachexia) & 6 of 69 \\
Blindness & 6 of 64 \\
Moderate to severe visual defects & 36 of 64 \\
Mild visual defects & 13 of 64 \\
Normal vision & 9 of 64 \\
\end{tabular}

STATISTICS

The duration of progression free survival was measured from the date of entry of the patient in the study to the date of progressive disease or last contact. Distributions of progression free survival and event free survival were estimated using the Kaplan-Meier method ${ }^{19}$ and comparisons were made using a Mantel-Haenzsel statistic. Standard errors in Kaplan-Meier estimates were calculated using Peto's technique. ${ }^{20}$

\section{Results}

CLINICAL PRESENTATION

Median age at diagnosis was 5 years (range, 3 months to 15 years), which was not significantly different between patients with or without NF1. All the children but one were symptomatic at the time of diagnosis, mainly with signs of visual impairment. In one patient, the tumour was found on the CT scan during surveillance of NF1. Table 1 summarises the clinical presentation of the patients.

\section{HISTOLOGY}

Tumour specimens had been obtained at diagnosis in 27 patients. The reviewed histological diagnosis was juvenile pilocytic astrocytoma in 16 patients; non-pilocytic low grade astrocytoma in eight patients; and hamartoma, schwannoma, and neurinoma in one patient each.

RADIOLOGICAL FINDINGS AT DIAGNOSIS AND RESPONSE TO RADIOTHERAPY

Tumour staging according to the Dodge classification was type I in six, type II in 10, and type III in 53 patients. None of the patients had metastasis at diagnosis but extensive spinal explorations were not performed. Two patients had metastasis at time of relapse. Most of the significant decreases in tumour size were seen after the first year following radiation (data not shown). Just over half of the 51 patients for whom response to radiotherapy was available experienced at least an objective response (fig 1). The disease progressed in only three children within the first year after radiotherapy. No clear correlation was found between tumour shrinkage and visual improvement.

\section{OPHTHALMOLOGICAL EVALUATION AT DIAGNOSIS} AND VISUAL OUTCOME

Forty four patients were investigated fully, with visual acuity determination, visual field, and fundus examination. Only three children had a completely normal ophthalmological examination. Thirty four of 41 children with deficit(s)

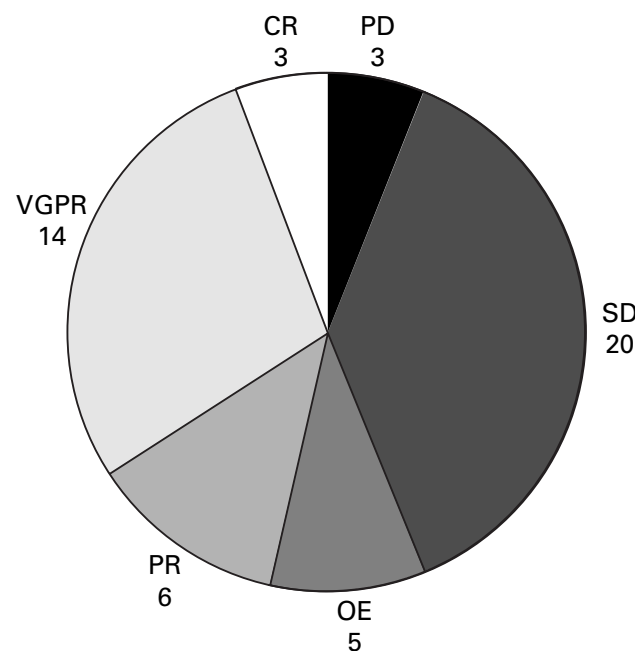

Figure 1 Best radiological response after radiation. Response assessed according to SIOP recommendations. ${ }^{18}$ $P D$, progressive disease; $S D$, stable disease; $O E$, objective response; $P R$, partial response; VGPR, very good partial response, $C R$ complete response.

had abnormal findings for both visual acuity, visual fields, and fundus. An abnormal visual field was present in all of the 41 children with a visual deficit except for one. Data on visual outcome were available for 62 patients. Four of the six patients who were blind at the time of diagnosis experienced no improvement, one had significant improvement, and one has been lost to follow up. Overall, 18 patients with initial visual defects had improved visual function after radiotherapy and 29 patients remained stable after treatment. Among nine patients with normal vision at diagnosis, six maintained normal vision without treatment and three maintained vision after radiotherapy. Thus, regardless of their initial visual capacity, 18 patients improved after radiotherapy; 29 patients remained stable with radiotherapy and eight without treatment; seven had visual deterioration, despite radiotherapy, even without a significant increase in tumour burden.

\section{ENDOCRINE EVALUATION AT DIAGNOSIS AND} AFTER TREATMENT

The two most frequent deficiencies at diagnosis were growth hormone deficiency and precocious puberty with low concentrations of follicle stimulating hormone ( $\mathrm{FSH})$ and luteinising hormone (LH). Table 2 summarises the endocrinological deficits present at diagnosis and the last follow up visit. Within a median follow up of 5 years (range, 6 months to 10 years), 47 patients had a complete endocrinological evaluation following treatment. Three had a normal endocrine status, 39 required permanent hormone replacement treatment, and five did not receive hormone replacement treatment despite a mild deficit.

SURVIVAL

The mean (SD) follow up of the patients included in this study was 7.6 (4.6) years, with a median follow up of 7 years. Initial treatment was surveillance in 16 , radiotherapy in 39 , and surgery in 14 patients. Overall survival of 
Table 2 Endocrine evaluations

\begin{tabular}{lcc}
\hline Hormone deficiency & $\begin{array}{l}\text { Before } \\
\text { treatment } \\
(n=29)\end{array}$ & $\begin{array}{l}\text { After } \\
\text { treatment } \\
(n=47)\end{array}$ \\
\hline $\begin{array}{l}\text { Growth hormone (GH) } \\
\text { Thyroid stimulting hormone (TSH) }\end{array}$ & 3 & 9 \\
GH and TSH & 1 & 2 \\
TSH and adrenocorticotrophic & 2 & 13 \\
$\quad$ hormone (ACTH) & 0 & 1 \\
Precocious puberty (PP) & 4 & 4 \\
GH and TSH and PP & 0 & 4 \\
GH and TSH and ACTH & 0 & 3 \\
GH and PP & 2 & 8 \\
Diabetes insipidus & 2 & 3 \\
Normal & 17 & 3 \\
\end{tabular}

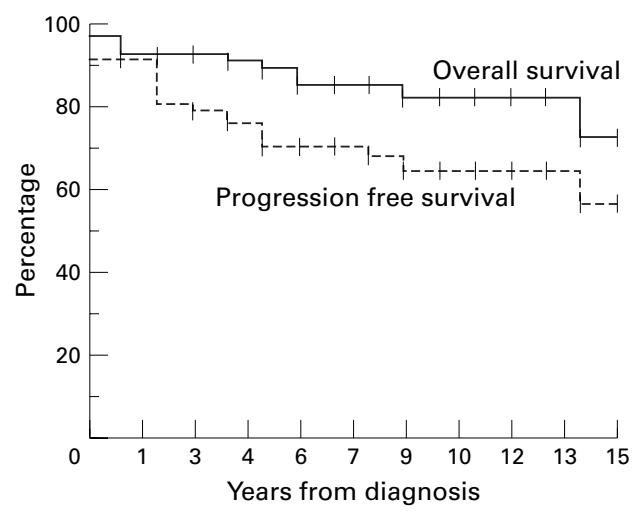

Figure 2 Survival curves of the 69 children in our study.

symptomatic patients with optic pathway gliomas continued to decrease after the fifth year of follow up, reaching $74 \%$ at 15 years (fig 2).

Overall and event free survival were better in children with NF1 than in the other children, but the difference was not significant (figs 3 and 4 ). The outcome of the patients according to the initial treatment is described in a flow diagram (fig 5). Among the six children with tumour confined to one optic nerve and with residual vision, three received irradiation initially and are long term survivors, and we decided on strict surveillance for the other three. Only one of them experienced progressive disease for which he received irradiation and this treatment has permitted long term progression free survival. Causes of death were progressive disease in seven, a secondary tumour in two, and a stroke in one patient. Of the two patients who died of a second tumour, one developed a Ewing's sarcoma outside the radiation field and the other had a progressive glioblastoma multiforme that had arisen inside the radiation field, 6.5 years and 14 years, respectively, after the diagnosis of the optic pathway tumour. The latter patient did not have NF1 and the glioblastoma was not secondary to the transformation of his optic pathway tumour.

CEREBROVASCULAR COMPLICATIONS

Nine patients experienced cerebrovascular complications during follow up. The mean age of these patients at irradiation ( 5 years; range, 2-8 years) was not different from the mean age of the whole population. All of them received radiotherapy with median doses of $54 \mathrm{~Gy}$. Thus, nine of 54 of the patients treated with irradiation experienced a cerebrovascular com-

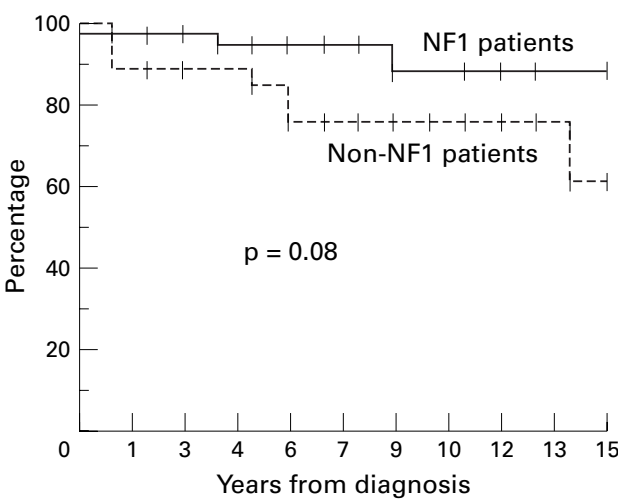

Figure 3 Overall survival of the 69 children in our study. Outcome of the children was analysed according to the presence or absence of NF1. The start point of the curve is diagnosis, whatever the initial treatment. When the start point of the analysis was the date of radiotherapy, the difference in overall survival was also not significant.

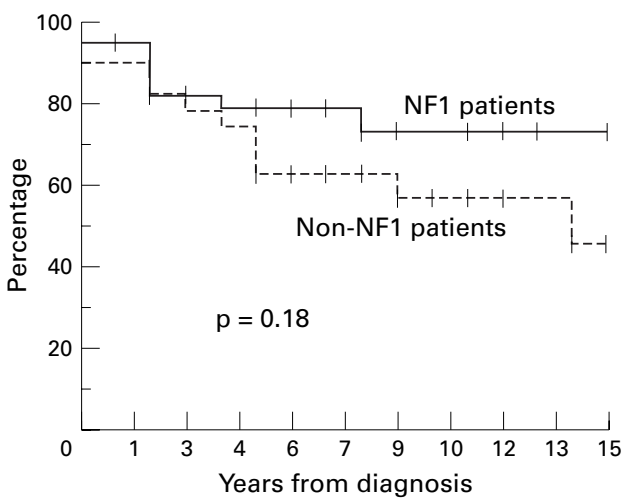

Figure 4 Progression free survival of the 69 children in our study. Outcome of the children was analysed according to the presence or absence of NF1. The start point of the curve is diagnosis, whatever the initial treatment. When the start point of the analysis was the date of radiotherapy, the difference in progression free survival was also not significant.

plication during follow up. Eight of them had NF1. Therefore, nine of 31 of the NF1 patients treated with irradiation experienced such a complication compared with only one of 22 patients without NF1. There was a median interval of 2.5 years between radiotherapy and the development of cerebrovascular complications (range, 6 months to 6 years). All but one were symptomatic. In this latter patient, a small ischaemic lesion was discovered in the internal capsule at reassessment of follow up scans. Two other patients had a transient ischaemic attack. Six patients experienced a stroke followed by hemiplegia. In three patients, angiography has been performed and showed stenosis of the carotid arteries and moya-moya vessels. One patient died of complications shortly after the stroke.

INTELLECTUAL OUTCOME

At the time of the last visit, 33 children had achieved a normal scholastic level commensurate with their age category (that is, $<2$ years behind), 10 of them attending a special school for blind children. Eighteen patients experienced major scholastic failure, 12 of them being placed in an institution for mentally retarded children. All of these severely disabled 


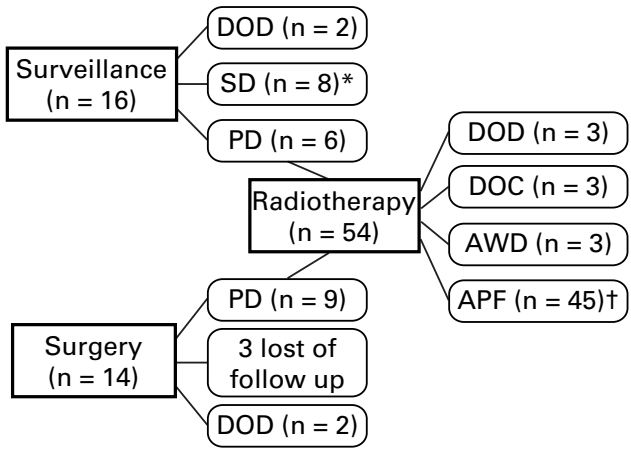

Figure 5 Outcome according to the initial treatment; number of patients is given for each subgroup. The 54 patients treated with radiotherapy were treated either initially $(n=39)$, after surgery $(n=9)$ or after initial surveillance $(n=6)$. *Sixteen patients (including 11 patients with neurofibromatosis type 1) were followed up initially without treatment; disease remained stable in eight of these patients during long term follow up (median, 4.5 years; range, 2-11); seven out of these eight had NF1. tRadiation doses ranged between 50 and $55 \mathrm{~Gy}$ in all the patients but two, who received doses lower than 40 Gy and subsequently relapsed. Forty five of the 54 patients treated with radiotherapy are alive without progression with a median follow up of 7 years (range, 5 months to 15 years). $P D$, progressive disease; $S D$, stable disease; $D O D$, death from disease; $D O C$, death from other cause; $A W D$, alive with disease on treatment; $A P F$, alive progression free.

children received radiotherapy, at a median age of 4 years. Intellectual outcome could not be assessed accurately and thoroughly in the 22 other children because of death $(n=11)$ or insufficient follow up $(n=11)$.

\section{Discussion}

Treatment regimens for optic pathway tumours are still a matter of debate. To clarify the effectiveness of conservative surgery and radiotherapy, we chose to analyse retrospectively a large number of patients treated during an era when modern imaging was available yet the current strategy of using chemotherapy had not been implemented. In this study, radiotherapy preceded or not by conservative surgery is shown to be a valuable treatment in terms of tumour control and visual preservation. However, in young children and in those with NF1, this approach should be reconsidered because of severe late effects. Once the tumour has invaded the chiasm, the optic tracts, or the diencephalon, surgical resection is generally no longer feasible. When confronted with clear cut tumour progression and visual impairment, radiotherapy has, until recently, been considered the standard treatment. Because most of the patients in our series presented with Dodge type II or III tumours, our treatment strategies were either based on surveillance or radiotherapy preceded by conservative surgery or not. The study and follow up of this cohort has helped us to define long term survival and morbidity for children with symptomatic optic pathway tumours. In our series, five year and 10 year overall survival rates were $91 \%$ and $83 \%$, respectively. Five year overall survival for patients with optic pathway tumours is $~ 85 \%$ in most studies, ${ }^{6}$ but this figure continues to decline with time. ${ }^{2}{ }^{21}$ Six late deaths occurred after the fifth year from diagnosis (two second tumours, three late relapses, and one complica- tion related death). This emphasises the need for prolonged follow up for proper evaluation of treatment strategies in these patients. There is a dearth of consistent information regarding the impact of radiation treatment on visual outcome. In addition, the reporting of visual outcome after radiotherapy has been largely unreliable because very young children are frequently considered unevaluable. However, our study and similar reports ${ }^{721}$ consider radiotherapy beneficial to visual function. Only a small proportion of children experienced a worsening of their visual status but almost a third experienced an improvement.

The fact that all the patients in our study were symptomatic with tumour progression at diagnosis led us to consider stable disease (both radiologically and clinically) as a yardstick of successful radiotherapy. However, among the 16 children who received no treatment, disease did not progress further during follow up in half of them. Thus, for some selected patients, aggressive treatment might legitimately be postponed, even when disease is symptomatic. Although a more indolent behaviour of optic pathway glioma has been suggested in patients with $\mathrm{NF} 1,{ }^{62}$ we cannot recommend surveillance as a rule for all patients with NF1 and optic pathway glioma. Because all of the children were followed up by CT scans and/or MRI, the radiological response to radiotherapy could be determined precisely and meticulously. More than half of the children who underwent irradiation achieved a significant radiological response, which became evident after the sixth month following radiotherapy.

Only a few studies (with a small number of patients) have reported the effect of radiotherapy on tumour size; response rates were similar to those obtained in our study. ${ }^{23}$ When the impact of chemotherapy or radiotherapy on optic pathway glioma is analysed in further trials, it should be borne in mind that radiological responses might be delayed and that children with stable disease at the end of radiotherapy might be cured, as was the case for almost half of our patients. In comparison, the best response rate with chemotherapy in newly diagnosed young patients reaches 57\% of significant radiological responses with carboplatin and vincristine. ${ }^{14}$ The relapse rate is low in patients treated with radiotherapy, ${ }^{24}$ as confirmed in this large series. Indeed, relapse is seen in $10-15 \%$ of patients who have undergone radiotherapy. $^{78}$ However, late tumour recurrences might be encountered after the fifth year, as was the case in four of our patients.

Recently, the presence of NF1 has been shown to be associated significantly with a lower risk of first relapse. ${ }^{25}$ This aspect could not be explored in our patients, because of the low relapse rate after radiotherapy. Moreover, as some children with NF1 did not receive radiotherapy initially and subsequently relapsed, we can assume that, once treated with radiotherapy, relapse free survival would also have been significantly higher in these patients. However, overall survival was not different for 
children with NF1 because many of them experienced treatment related adverse effects or second tumours.

Complications currently reported in children receiving radiotherapy for optic pathway tumours concern impaired cognitive development $^{9}$ and hormone deficiency. ${ }^{15}$ Among the 47 children who had a complete endocrinological evaluation after radiotherapy, only three had a normal endocrine status. Of all the pituitary hormones, growth hormone was the most sensitive to radiotherapy because deficiency of this hormone was seen in 37 of the 44 patients with hormone deficiency. In agreement with other reports, the intellectual outcome was poor for those patients in our series who received radiotherapy, especially before the age of 4 years. This emphasises the need for careful neuropsychological surveillance in children receiving radiotherapy for optic pathway gliomas, so that an adapted rehabilitation programme can be planned. There are now increasing case reports of cerebrovascular complications after radiotherapy for optic pathway glioma. Among 40 patients with radiation related cerebral occlusive disease reported elsewhere, 16 received radiotherapy for optic pathway gliomas. ${ }^{26}$ However, the frequency of this complication was not determined precisely in a large series of patients submitted to radiotherapy for optic pathway glioma. To date (and this figure may increase with prolonged follow up), almost a fifth of the children have experienced complications of radiation induced vasculopathy. All but one had NF1. Underlying vascular abnormalities in these patients might exacerbate the risk of such complications, as suggested in one report. ${ }^{27} \mathrm{With}$ more patients with NF1 being treated with irradiation, our study shows that this complication is five times more frequent in patients with NF1. Cerebrovascular complications occurred early during follow up in our study. However, as reported by Bitzer et $a l,{ }^{26}$ this complication can sometimes occur late after irradiation - that is, 10 years after treatment. Because of the high rate of radiation related complications, we propose delaying radiotherapy in patients with NF1 if possible, and suggest that other therapeutic options should be considered first. If irradiation is performed, longitudinal follow up of the cerebral vessels should be included in the surveillance from the first year after treatment.

\section{Conclusion}

For progressive optic pathway gliomas involving the optic chiasm or diencephalon, radiotherapy with or without conservative surgery is a valuable treatment strategy in terms of tumour response, visual outcome, and progression free survival. However, treatment related complications can be unacceptable, especially in patients with NF1 who are at risk for radia- tion induced cerebral vasculopathy. Therefore, radiotherapy is no longer recommended as first line treatment in children younger than 5 years or those suffering from NF1. The role of chemotherapy with or without conservative surgery should be explored in these patients at higher risk for radiation related complications.

We thank Ms Lorna Saint-Ange for editing the manuscript.

1 Heideman RL, Freeman CR, Packer RJ, Rorke LB, Albright LA. Tumors of the central nervous system. In: Pizzo PA and Poplack DG, eds. Principles and practice of pediatric oncology. Poplack DG, eds. Principles and practice of pedia

2 Chang CH, Wood EH. The value of radiation therapy for gliomas of the anterior visual pathway. In: Brockhurst RJ, Borouchoff SA, Hutchinson BT, eds. Controversy in ophthalmology. Philadelphia: WB Saunders, 1977:878-86.

3 Gajiar A, Bhargava R, Jenkins JJ, et al. Low-grade astrocytoma with neuraxis dissemination at diagnosis. $\mathcal{F}$ Neurosurg 1995;83:67-71.

4 Bruggers CS, Friedman HS, Phillips PC, et al. Leptomeningeal dissemination of optic pathway gliomas in three children. Am f Ophthalmol 1991;111:719-23.

5 Hoffman HJ, Humphreys RP, Drake JM, Rutka JT, Jenkin D, Greenberg M. Optic pathway/hypothalamic gliomas: a dilemma in management. Pediatr Neurosurg 1993;19:18695.

6 Garvey M, Packer RJ. An integrated approach to the treatment of chiasmatic-hypothalamic gliomas. $\mathcal{f}$ Neurotreatment of chiasma $1996 ; 28: 167-83$.

7 Kovalic JJ, Grigsby PW, Shepard MJ, Fineberg BB, Thomas PR. Radiation therapy for glioma of the optic nerve and chiasm. Int f Radiat Oncol Biol Phys 1990;18:927-32.

8 Pierce SM, Barnes PD, Loeffler JS, McGinn C, Tarbell NJ. Definitive radiation therapy in the management of symptomatic patients with optic glioma. Cancer 1990;65:45-52.

9 Packer RJ, Savino PJ, Bilaniuk LT, et al. Chiasmatic gliomas of childhood: a reappraisal of natural history and effectiveness of cranial radiation. Child's Brain 1983;10:393-403.

10 Rosenstock JG, Packer RJ, Bilaniuk I, Bruce DA, Radcliffe J, Savino P. Chiasmatic optic gliomas treated with chemotherapy. $\mathcal{F}$ Neurosurg 1985;63:862-6.

11 Petronio J, Edwards MSB, Prados M, et al. Management of chiasmal and hypothalamic gliomas of infancy and childhood with chemotherapy. I Neurosurg 1991;74:701-8.

12 Kalifa C, Raquin MA, Plantaz D. Chemotherapy for low-grade gliomas. Med Pediatr Oncol 1995;25:246.

13 Prados MD, Edwards MS, Rabbitt J, Lamborn K, Davis Prados MD, Edwards MS, Rabbitt J, Lamborn K, Davis
RL, Levin VA. Treatment of pediatric low-grade gliomas $\mathrm{RL}$, Levin VA. Treatment of pediatric low-grade gliomas
with a nitrosourea-based multiagent chemotherapy regiwith a nitrosourea-based multiagent
men. $₹$ Neurooncol $1997 ; 2: 235-241$.

14 Packer RJ, Ater J, Allen J, et al. Carboplatin and vincristin chemotherapy for children with newly diagnosed progressive low-grade gliomas. F Neurosurg 1997;86:747-54.

15 Brauner R, Malandry F, Rappaport R, et al. Growth and endocrine disorders in optic glioma. Eur $\mathcal{F}$ Pediatr 1990;149:825-8.

16 National Institute of Health consensus development conference. Neurofibromatosis: conference statement. Arch Neurol 1988;45:575-8.

17 Dodge HW, Lowe JG, Craig WM, et al. Gliomas of the optic nerves. Archives of Neurology and Psychiatry 1958;79:60721.

18 Gnekow AK. Recommendations of the brain tumor subcommittee for the reporting of trials. Med Pediatr Oncol 1995;24:104-8.

19 Kaplan EL, Meier P. Nonparametric estimation from incomplete observations. 7 Am Stat Assoc 1975;53:457-81.

20 Peto R, Pike MC, Armitage P. Design and analysis of randomised clinical trials requiring prolonged observation randomised clinical trials requiring prolonged

21 Jenkin D, Angyalfi S, Becker L, et al. Optic glioma in children: surveillance, resection, or irradiation? Int $\mathcal{F}$ Radiat Oncol Biol Phys 1993;25:215-25.

22 Listernick R, Charrow J, Greenwald M, Mets M. Natural history of optic pathway tumors in children with neurofibromatosis type 1: a longitudinal study. I Pediatr 1994;125: 63-6.

23 Fletcher WA, Imes RK, Hoyt WF. Chiasmal gliomas: appearance and long-term changes demonstrated by computerized tomography. 7 Neurosurg $1986 ; 65: 154-9$.

24 Alvord EC, Lofton S. Gliomas of the optic nerve or chiasm. f Neurosurg 1988;68:85-98.

25 Deliganis AV, Geyer JR, Berger MS. Prognostic significance of type 1 neurofibromatosis (von Recklinghausen disease) of type 1 neurofibromatosis (von Recklinghausen disease)

26 Bitzer M, Topka H. Progressive cerebral occlusive disease after radiation therapy. Stroke 1995;26:131-6.

27 Kestle JR, Hoffmann HJ, Mock AR. Moyamoya phenomenon after radiation for optic glioma. $\mathcal{F}$ Neurosurg 1993;79: $32-5$ 